\title{
Atomic force microscopy study of the growth and annealing of Ge islands on $\mathrm{Si}(\mathbf{1 0 0})$
}

Bing Liu, Cindy L. Berrie, Takeshi Kitajima, John Bright, and Stephen R. Leone ${ }^{a)}$

JILA, University of Colorado and National Institute of Standards and Technology, and Department of Chemistry and Biochemistry and Department of Physics, University of Colorado, Boulder, Colorado 803090440

(Received 24 October 2001; accepted for publication 21 January 2002)

\begin{abstract}
Atomic force microscopy is used to study the growth and annealing of $\mathrm{Ge}$ islands on $\mathrm{Si}(100)$ by molecular beam epitaxy. The $\mathrm{Ge}$ island shape, size distribution, number density, and spatial distribution under various growth conditions, such as different substrate temperatures, Ge beam fluxes, and annealing times, are investigated. By limiting the growth to a low coverage of $6 \mathrm{ML}$ of $\mathrm{Ge}$, we find that either a low growth temperature $(\leqslant 875 \mathrm{~K})$ or a high beam flux can produce films dominated by pyramids of $\{105\}$ facets. Domes of higher aspect ratios only appear at high growth temperatures or after a long time of annealing at low temperatures. This indicates that in the competition between the different kinetic processes responsible for the pyramid and dome formation, the domes require a higher activation energy and grow slower. We also demonstrate that appropriate annealing at low temperature can form locally ordered arrays of pyramids with a narrow size distribution. (C) 2002 American Vacuum Society. [DOI: 10.1116/1.1459724]
\end{abstract}

\section{INTRODUCTION}

Growth of $\mathrm{Ge}$ on $\mathrm{Si}$ is a classical example of the Stranski-Krastanov (SK) growth mode, i.e., a wetting layer of several monolayer (ML) thickness [3-5 $\mathrm{ML}$ for $\mathrm{Ge}$ / $\mathrm{Si}(100)]$ is followed by formation of three-dimensional (3D) islands. Smaller than a certain size, the $3 \mathrm{D} \mathrm{Ge}$ islands on $\mathrm{Si}$ are coherently strained (dislocation free), ${ }^{1}$ and therefore the growth processes are potentially applicable to the fabrication of device-quality self-assembled quantum dots. In previous studies, ${ }^{2-4}$ two types of coherent Ge islands on $\mathrm{Si}(100)$ have been observed, differing in both size and shape. They are pyramids bounded by four $\{105\}$ facets, and domes of steeper and complex facets [e.g., $\{113\}$ and $\{102\}]$. During growth, $\mathrm{Ge}$ islands first appear as pyramids. When reaching a certain size, the pyramids change shape to become domes. ${ }^{5}$

In order to explain the bimodal size distribution and the shape transition of $\mathrm{Ge} / \mathrm{Si}(100)$ islands, Ross et al. ${ }^{5}$ studied the dependence of the chemical potential on island shape and size. They proposed that, at a certain size, the chemical potential of an island of a high aspect ratio becomes lower than that of an island of a low aspect ratio, and an island shape transition occurs as a first order phase transition. After the transition, similar to the case of the classical Ostwald ripening, the steeper islands will grow larger at the expense of the smaller and flatter islands.

Severāl processes have been suggested to contribute to the pyramid to dome transition. Comers of a pyramid could first start to dissolve because of their sharp curvatures. ${ }^{6}$ The atoms at the island edge could disassociate because of the high local strain. ${ }^{7,8} \mathrm{Si}-\mathrm{Ge}$ intermixing will also help to relieve the strain, $^{8-11}$ and as a result both types of islands obtain higher contents of $\mathrm{Si}$ and larger volumes. ${ }^{9}$

\footnotetext{
Author to whom correspondence should be addressed: srl@jila.colorado.edu; National Institute of Standards and Technology.
}

On the other hand, Kamins et al. ${ }^{12}$ (>900 K) observed that, during annealing of $\mathrm{Ge} / \mathrm{Si}(100)$ islands at high temperetures, a dome island could change its shape back to a pyremid. They suggested that both shapes (i.e., pyramid and dome) are stable structures for coherent $\mathrm{Ge} / \mathrm{Si}(100)$ islands.

For the fabrication of self-assembled quantum dots, it is desirable to have a narrow island size distribution. It is apparent that the bimodal size distribution of Ge islands on $\mathrm{Si}(100)$ is an impediment to this goal. It is worthwhile to study additional aspects of the growth kinetics of pyramids and domes, so that we may find appropriate conditions to control the growth to obtain a stable ensemble of islands of only one type and a narrow size distribution.

In the present work, using atomic force microscopy (AFM) and growth by molecular beam epitaxy (MBE), we first study the morphology dependence of Ge islands on $\mathrm{Si}(100)$ as a function of growth temperature and beam Differing from many previous studies of $\mathrm{Ge} / \mathrm{Si}(100)$ islands. our growth experiments are limited to a low coverage of 6 monolayers (MLs), which is just above the critical thickness for the SK growth of $\mathrm{Ge} / \mathrm{Si}(100)$. At higher coverngess lands can become large enough to relax dislocations. $^{13,14}$ A dislocated island will grow very through become a very large, relaxed "superdome" (named fast to iams et $a l^{15}$ ). Superdomes will not only consumed by Will. supersaturation, but also the surrounding mest of the islands. ${ }^{16}$ This complicates the interpretation small coherent sity and size distribution.

In the second part of this work, we study the effect of low temperature annealing on the morphology and 2D (lateral)
ordering of the islands. It has been sugg strain favors the formation of periodiggested that the film strain favors the formation of periodic domains ${ }^{17,18}$ (e.g. a im observed previously in InAs/GaAs heteroepitaxy 19,20 been ever, contradictory results were also obtained for this system. 


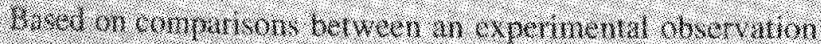

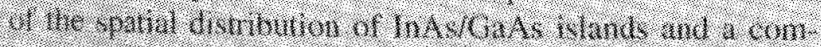

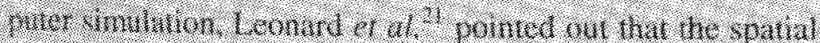

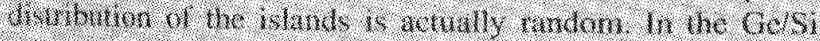

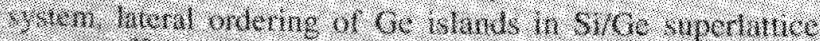

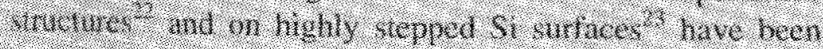

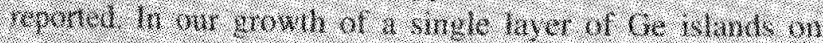

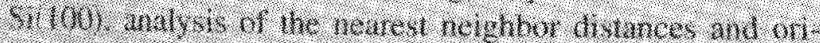

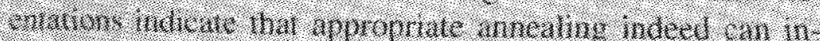

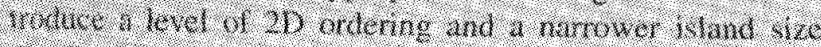
1.r.tunu

\section{EXPERWENT}

Al grown expermeni were perromed in a home buin UAF anwber with a base pressure in the $1 \times 10$. 10 Tom

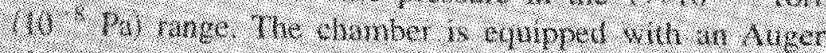

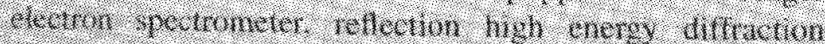

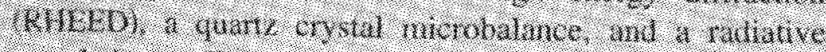

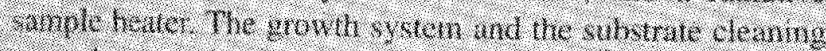

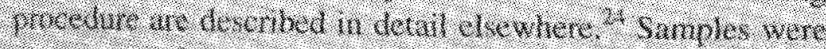

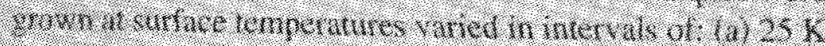

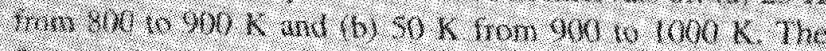

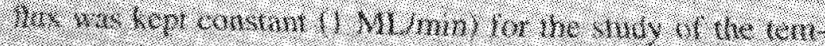

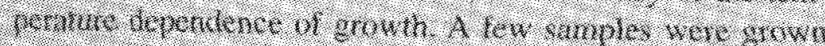

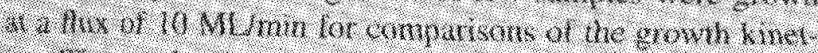

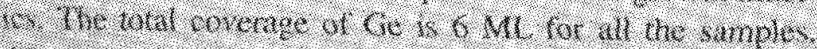

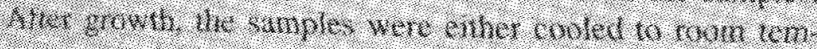

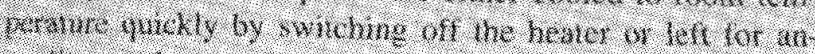

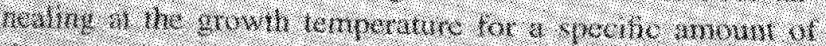
hise.

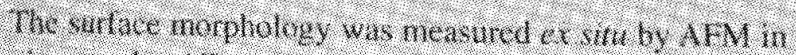

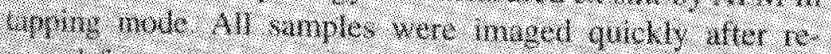

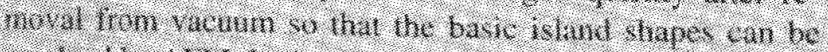

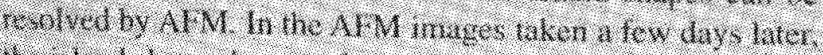

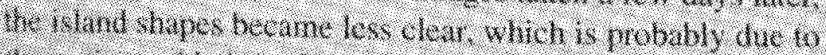

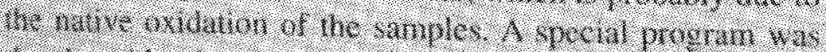

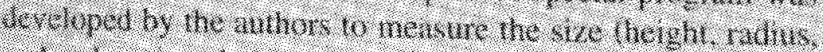

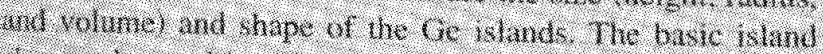

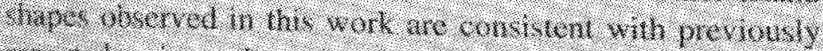
proted in ith scimmo imneling microbcopy

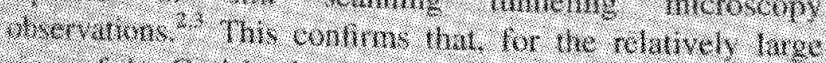

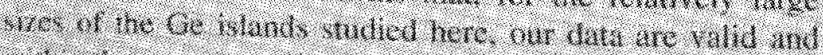

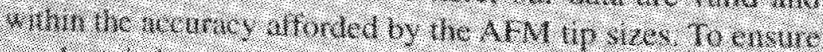

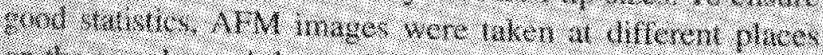

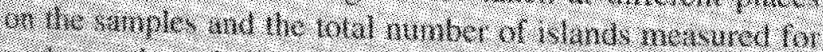

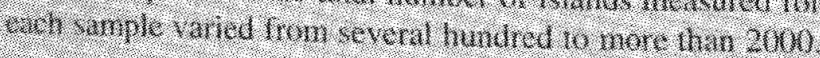

\section{RESULTS AND DISCUSSION}

\section{A. Crown of 6 ML of Ce on Si(100) at diverent tubstrate temperatures without annealing}

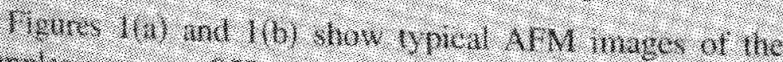

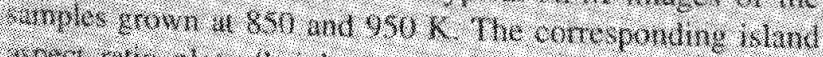

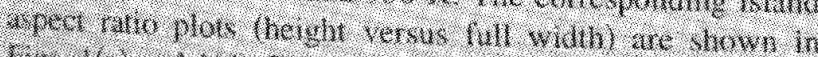

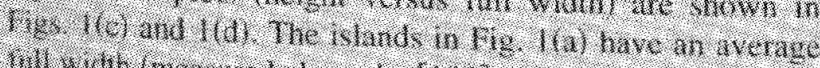

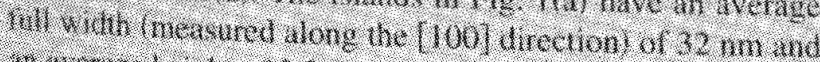

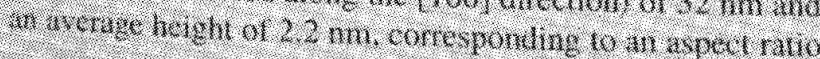

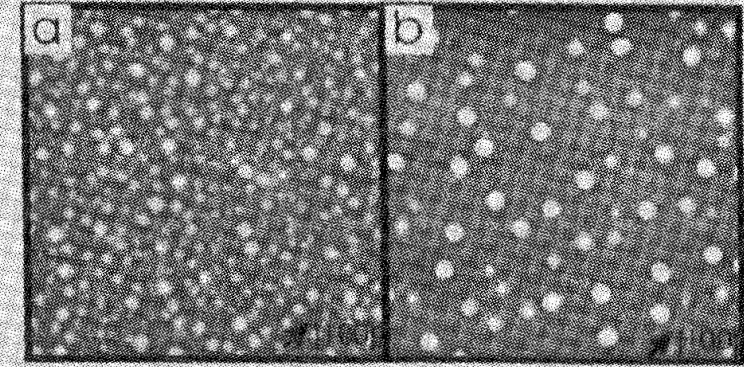

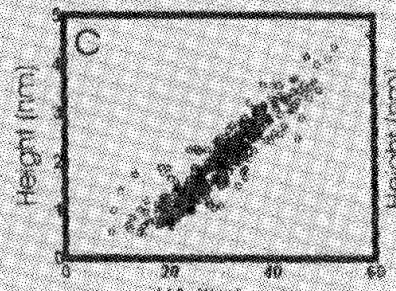

Watim (n)

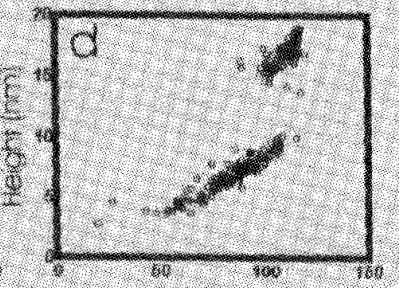

W.

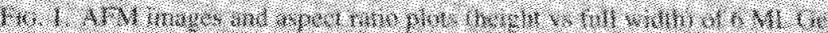

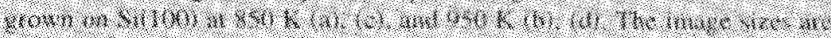

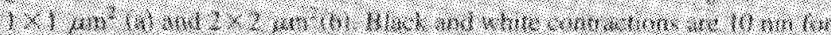

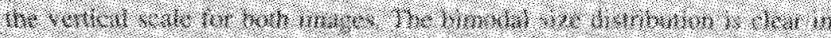

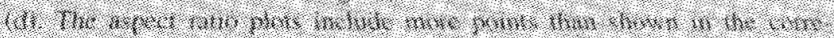

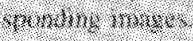

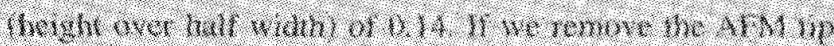

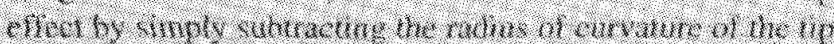

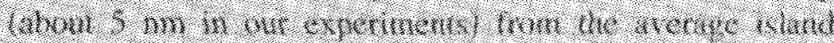

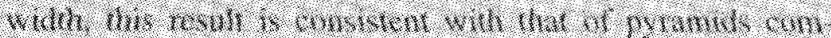

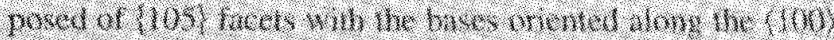

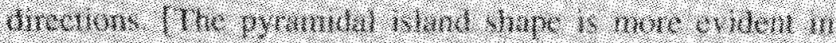

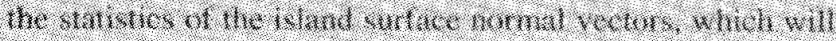

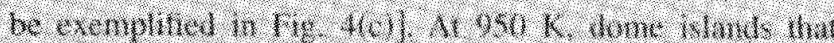

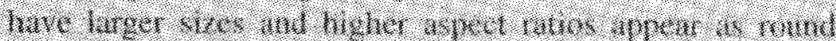

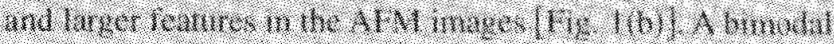

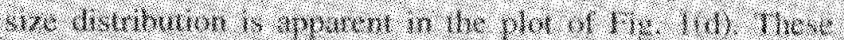

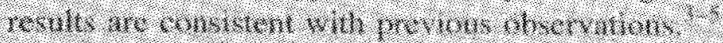

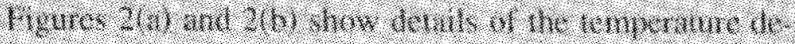

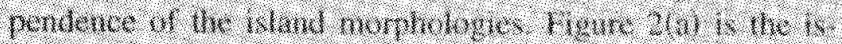

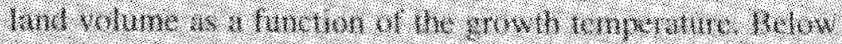

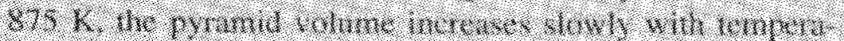

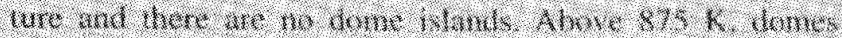

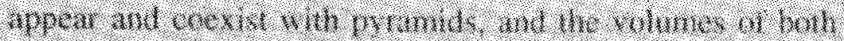

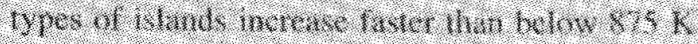

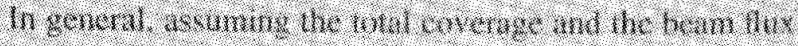

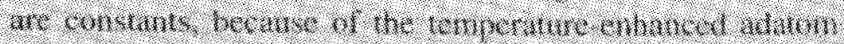

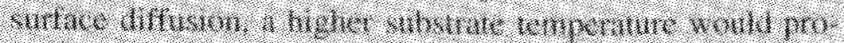

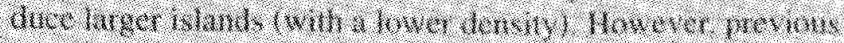

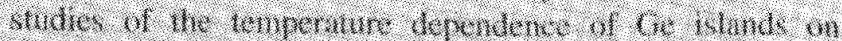

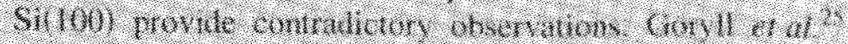

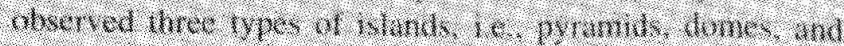

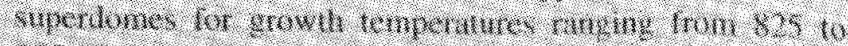

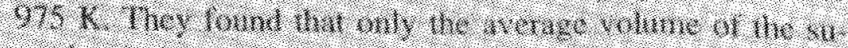

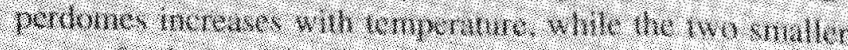

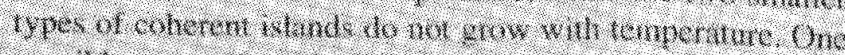

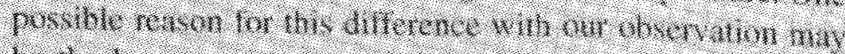

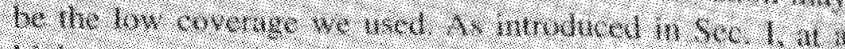

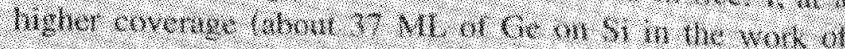



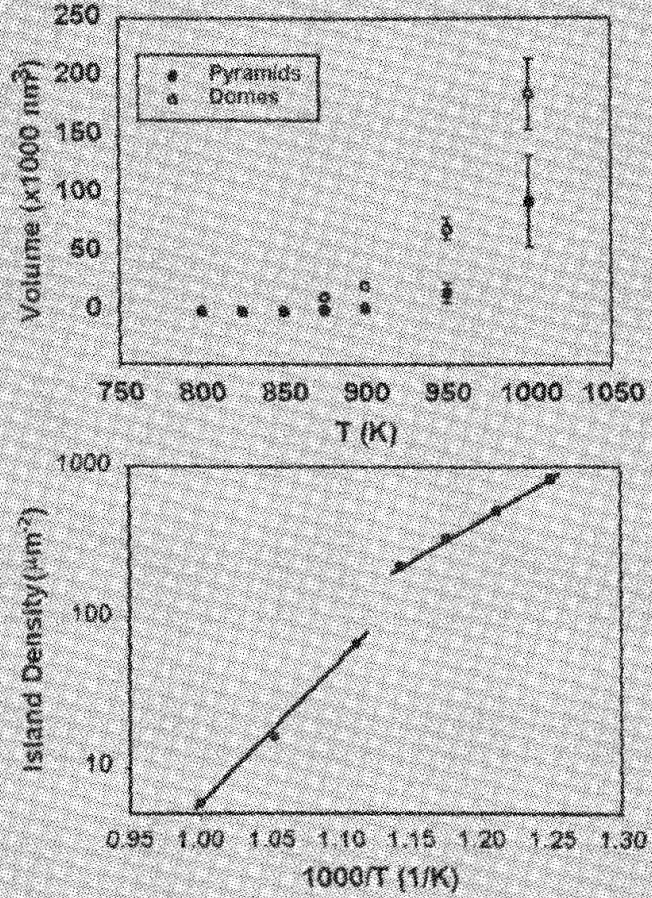

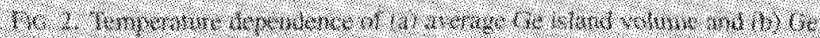

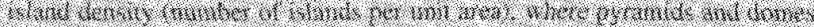

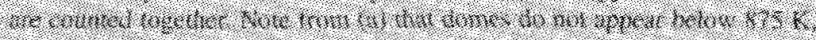

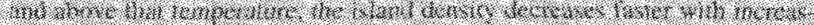

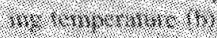

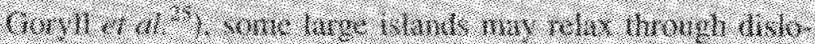

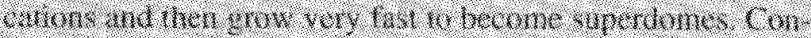

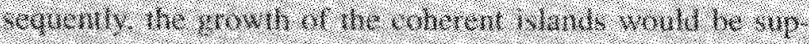

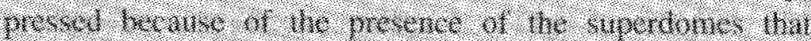

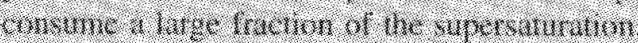

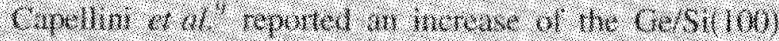

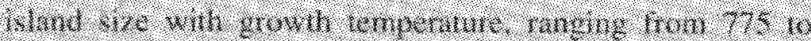

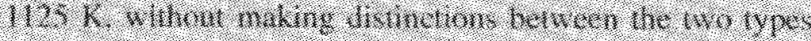

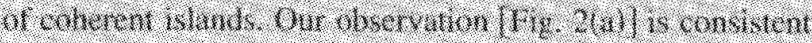

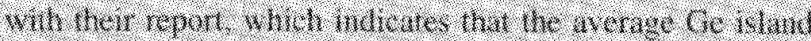

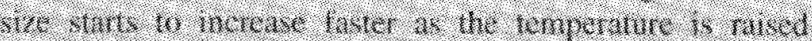

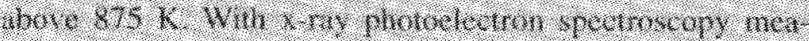

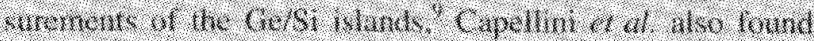

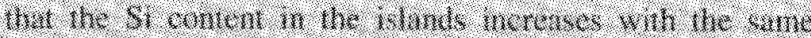

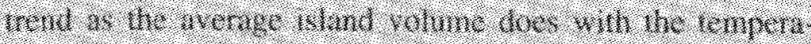
me Thi mems hat a aderren w be cheet of the

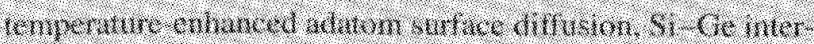

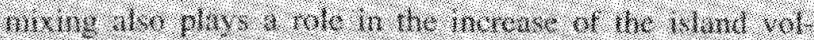
Wintes:

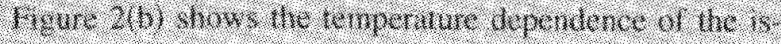

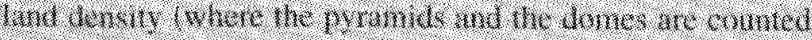

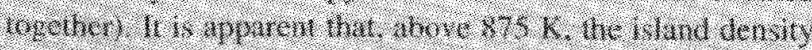

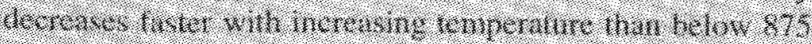

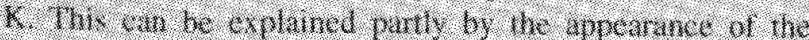

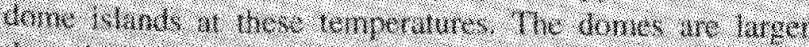

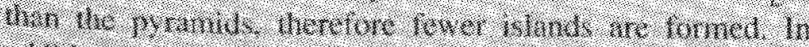

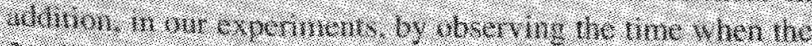

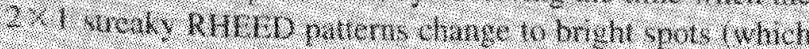

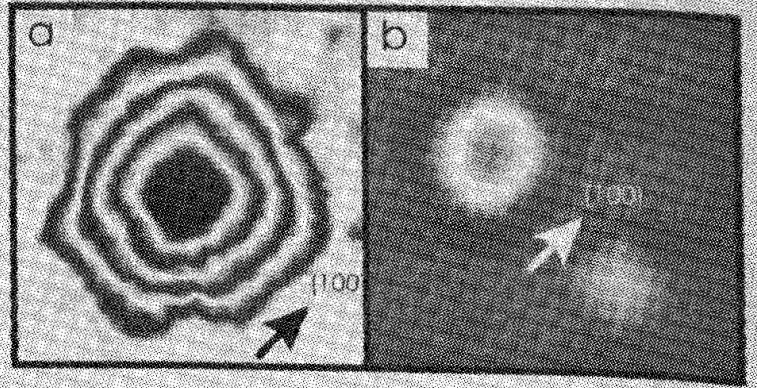

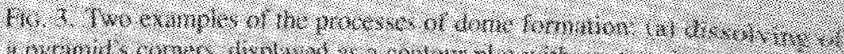

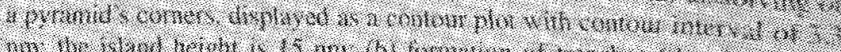

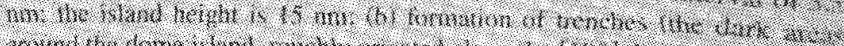

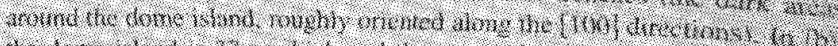

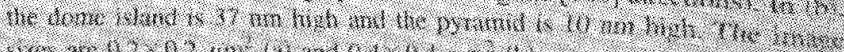

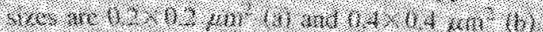

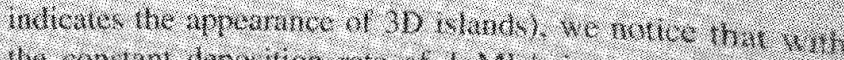

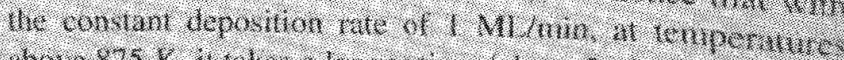

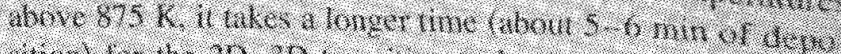

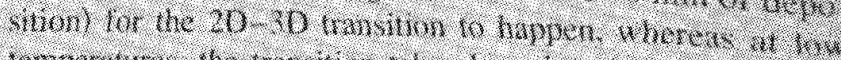

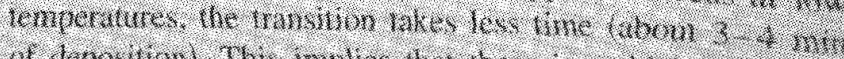

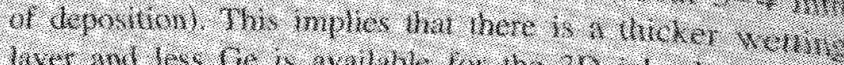

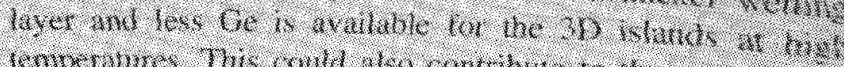

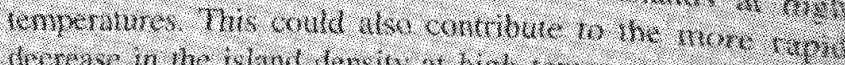

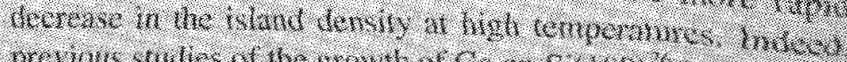

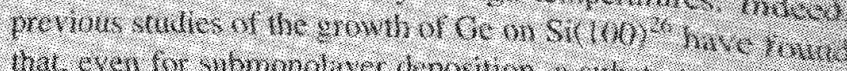

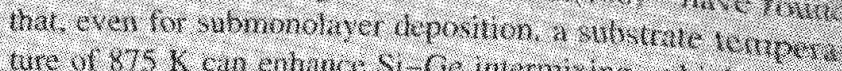

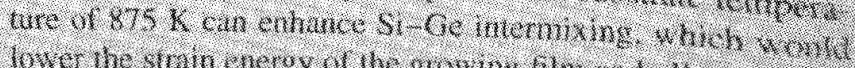

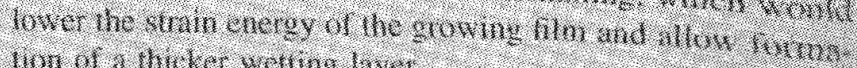
wor of a hither welling livet

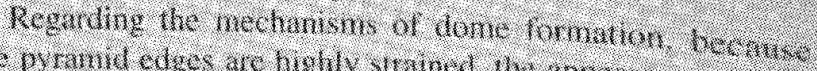

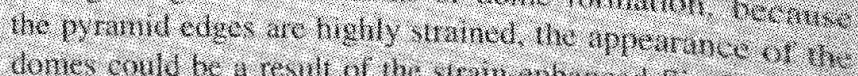

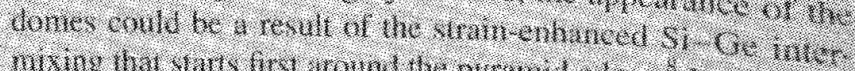

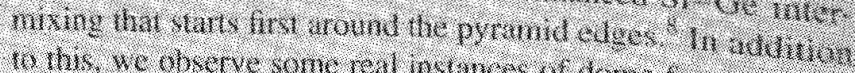

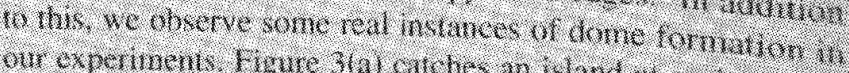

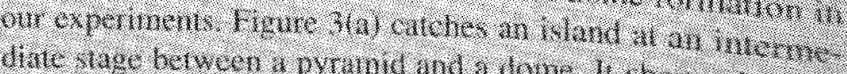

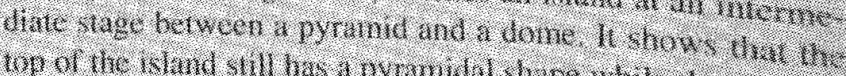

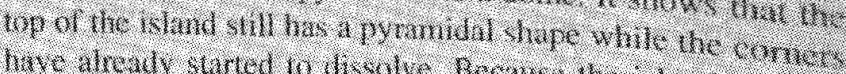

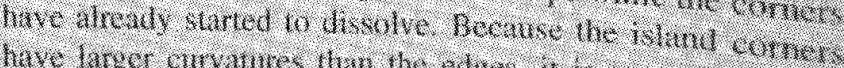

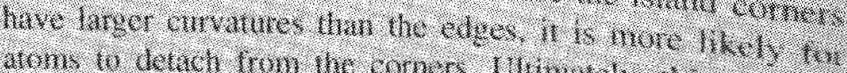

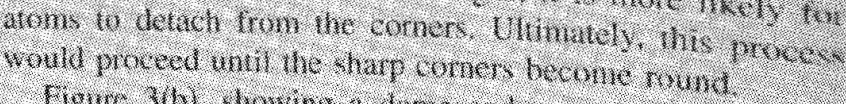

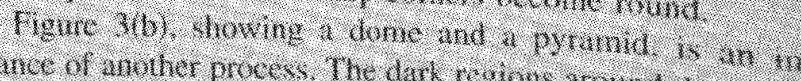

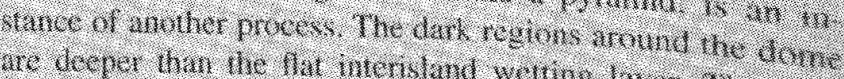

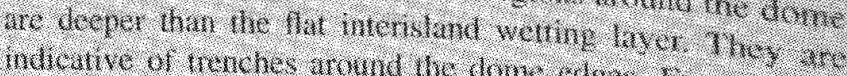

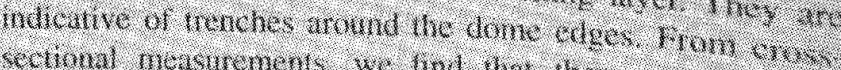

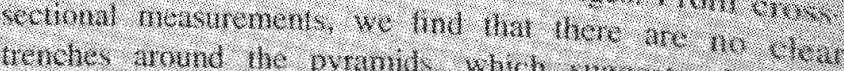

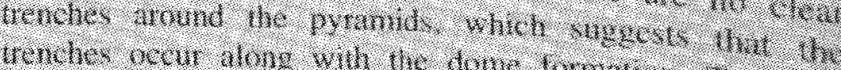

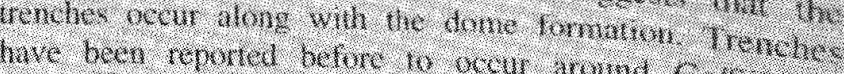

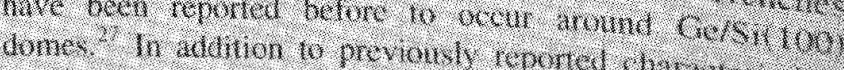

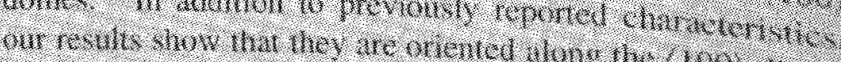

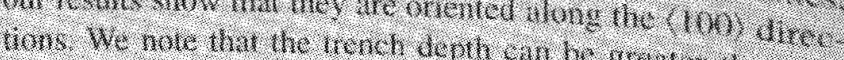

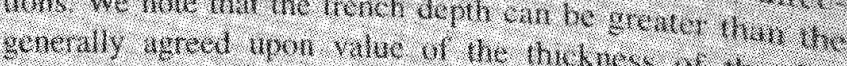

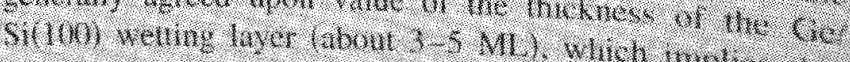

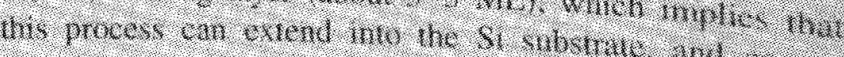

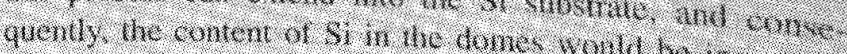



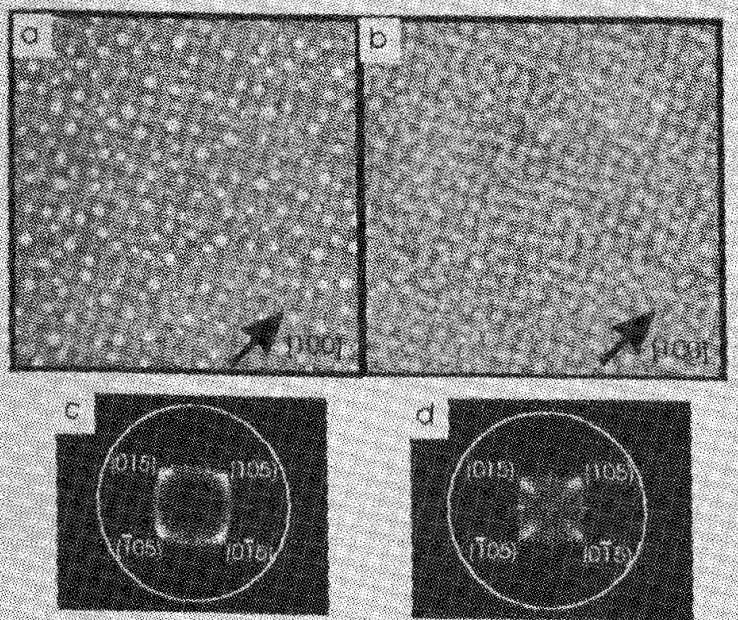

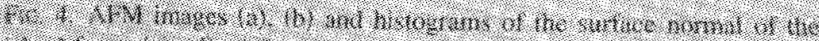

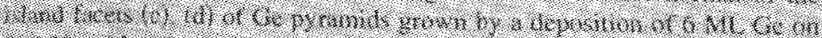

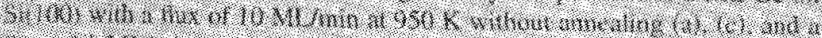

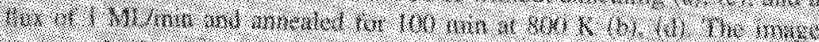

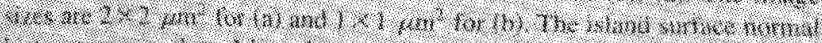

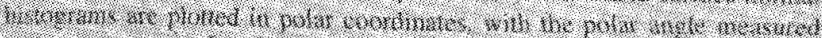

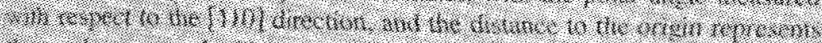

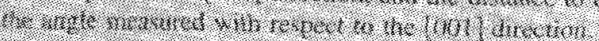

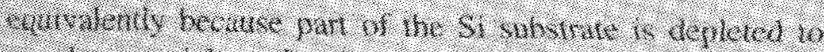

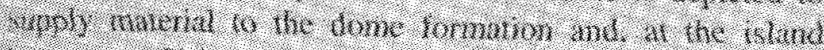

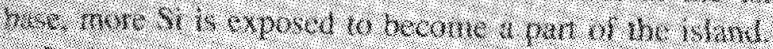

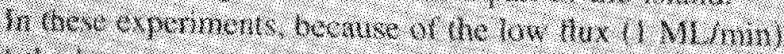

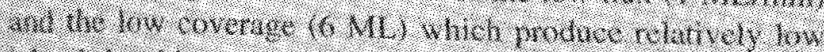

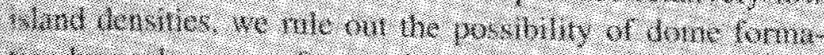

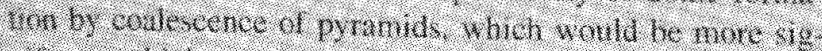

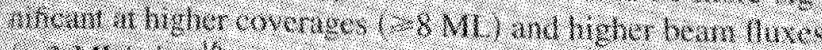
- Vhrint?

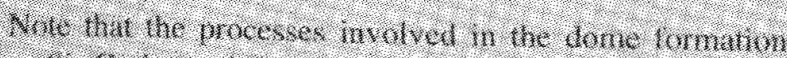

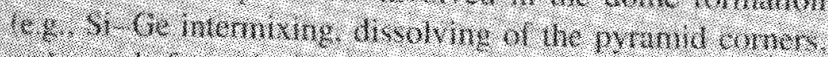

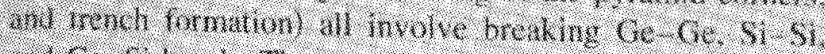

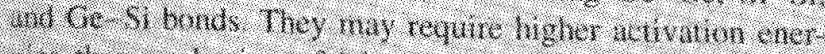

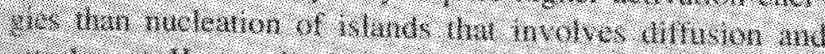

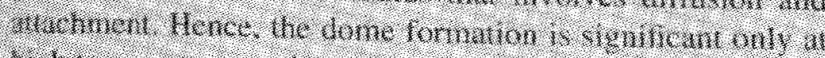

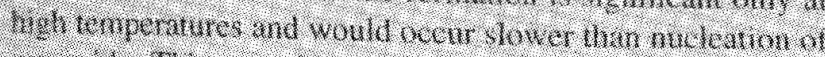

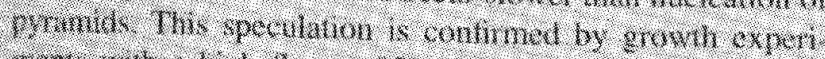

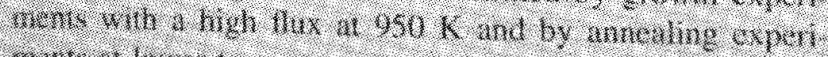

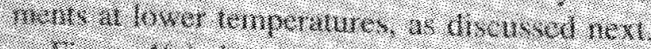

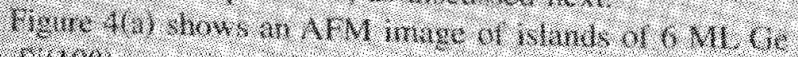

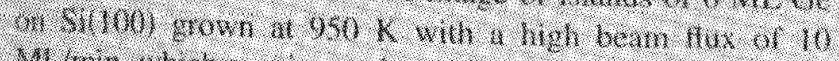

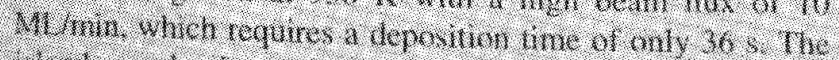

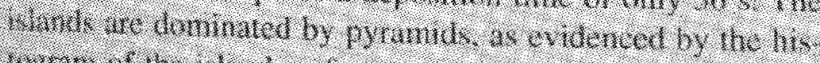

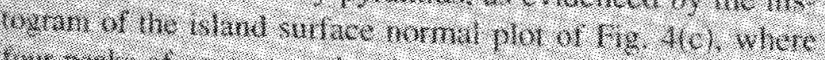

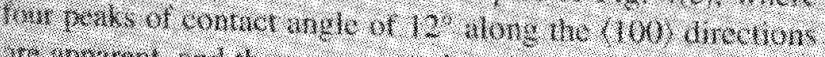

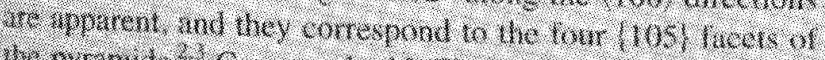

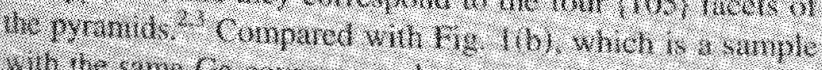

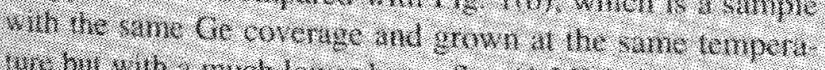

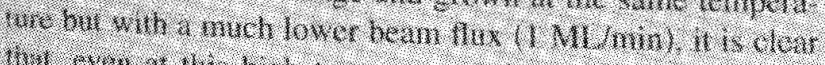

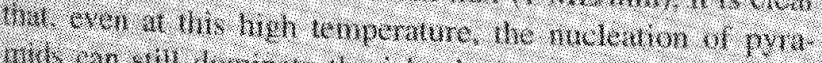

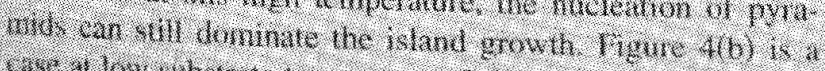

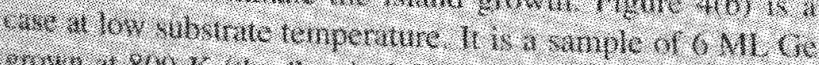

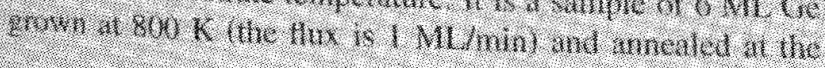

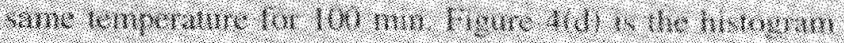

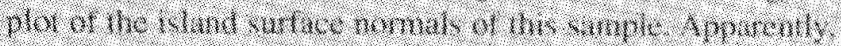

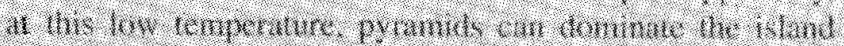

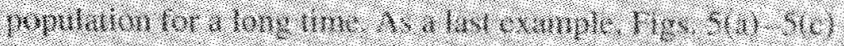

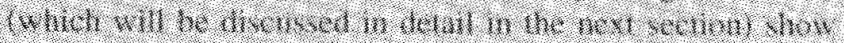

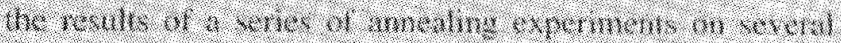

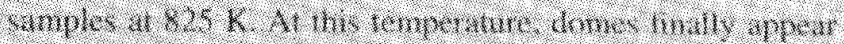

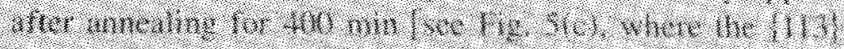

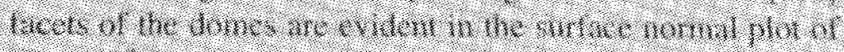
Iis. $5 \mid \pi$

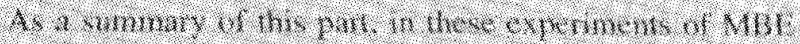

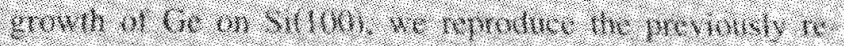

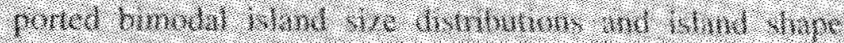

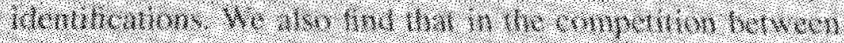

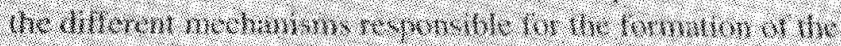

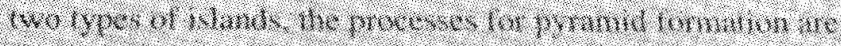

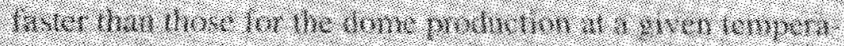

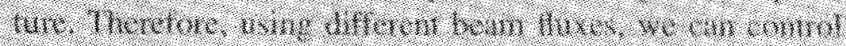

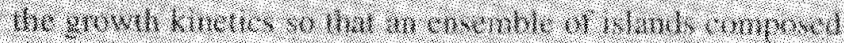

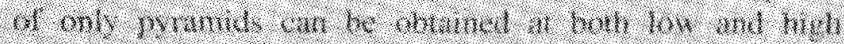

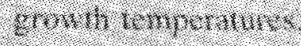

\section{B. Eftects of low temperature anewing on the istand morphology}

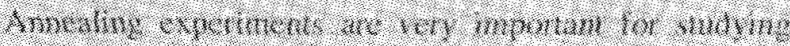

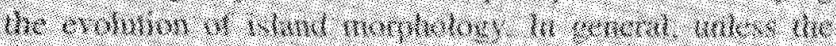

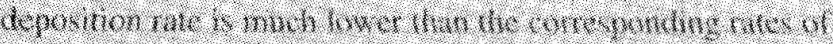

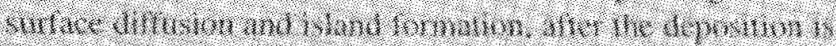

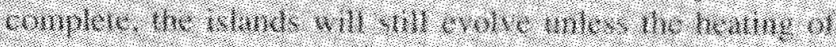

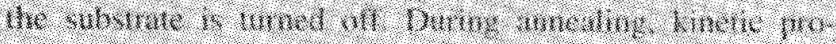

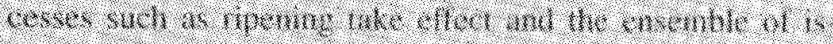

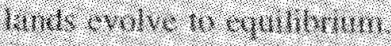

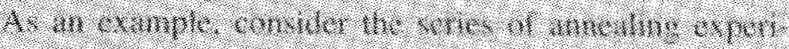

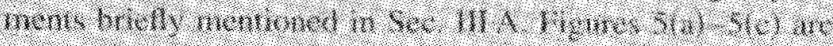

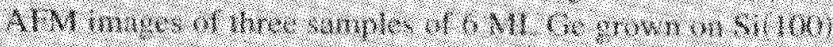

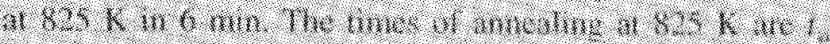

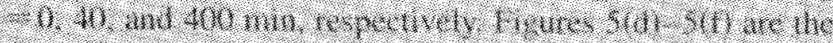

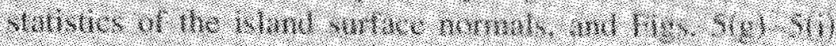

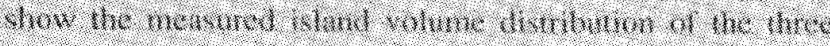

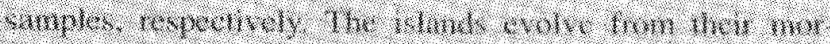

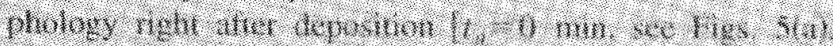

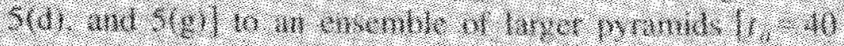

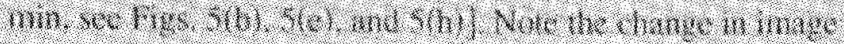

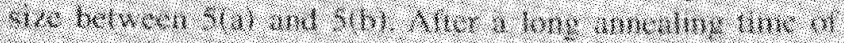

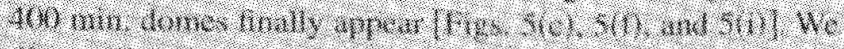

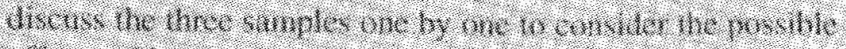

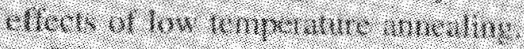

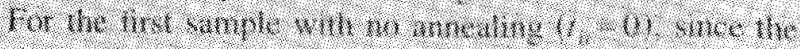

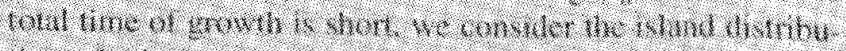

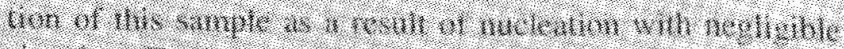

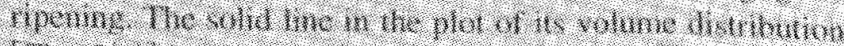

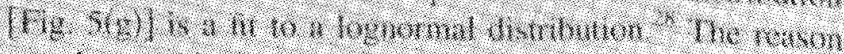

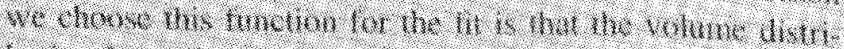

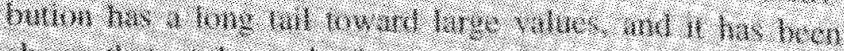

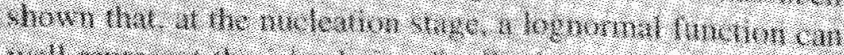

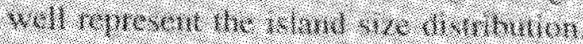



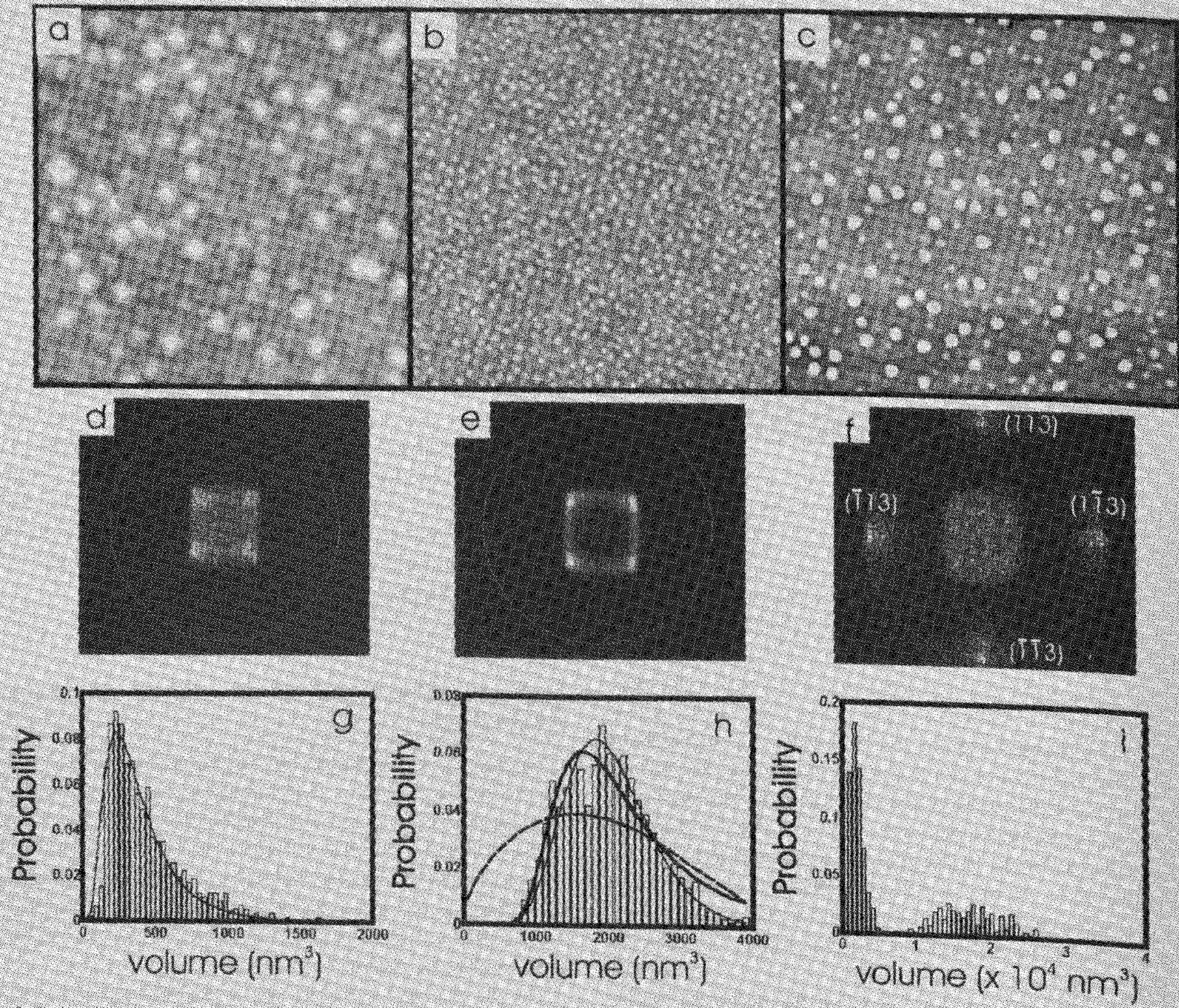

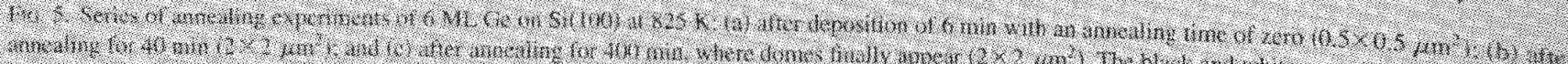

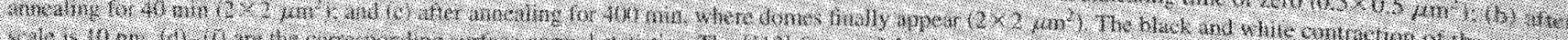

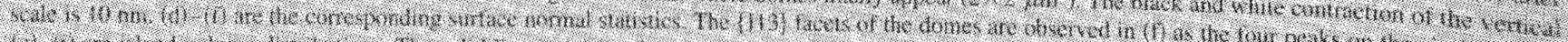

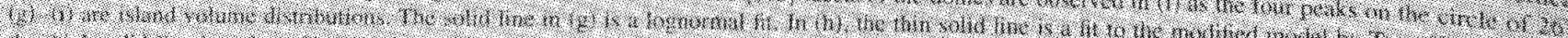

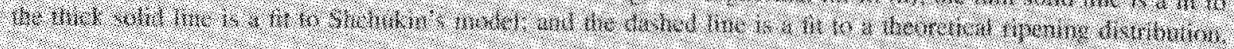

Now emoides the second sample with $t_{i}=40$ win. If is intercsing fo nate that for the sample, the isiands are fot only sill donmated by pyrands, but they also seen wo have more vinifrom size, shape, and spatal drstributions than without ansealing. The tathe of the standard deviation of island welume wer the nean island wolume for this smple is ahout

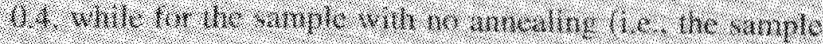

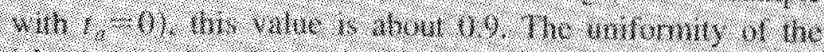
thand shape is reflected is the surface nomal statistic Fis. ure s(e) shows shirper peaks of the $\{105\}$ lacets than fig. S(10.

The spatial distrbition of the islinds of this sample is atso worth noting As intrialiced in Sec. 1 , it was predicted

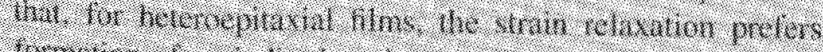
formation of periodic diomains lese arrays of islands). This

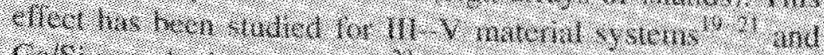

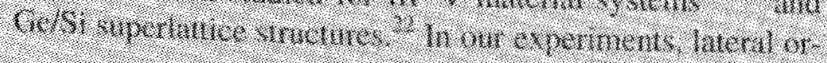

leving of the pyramids is ahserved for wis ample with ristes of the neareg of

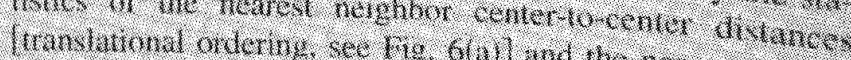

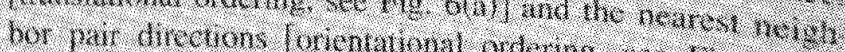
There is evidente that both probatilityms: see fige f(6) peak at di few values, surgestion tew of

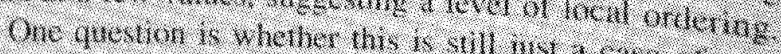
dom distribition of clusters, which could af cite of it ran

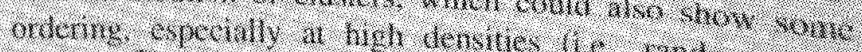

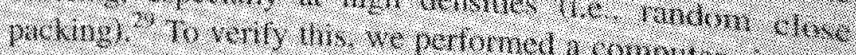
tion of a randon distribution of an 20 cluster. To ensure the sane zugh ensemble of the $2 \mathrm{D}$ cluster in the sineverse of whands. Whe

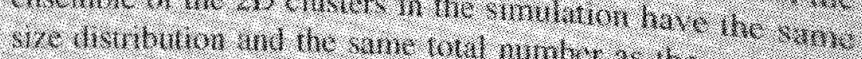

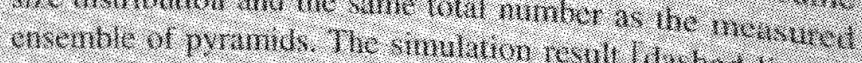

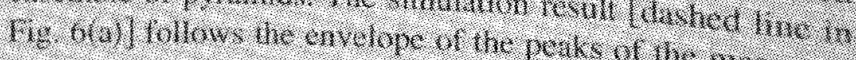



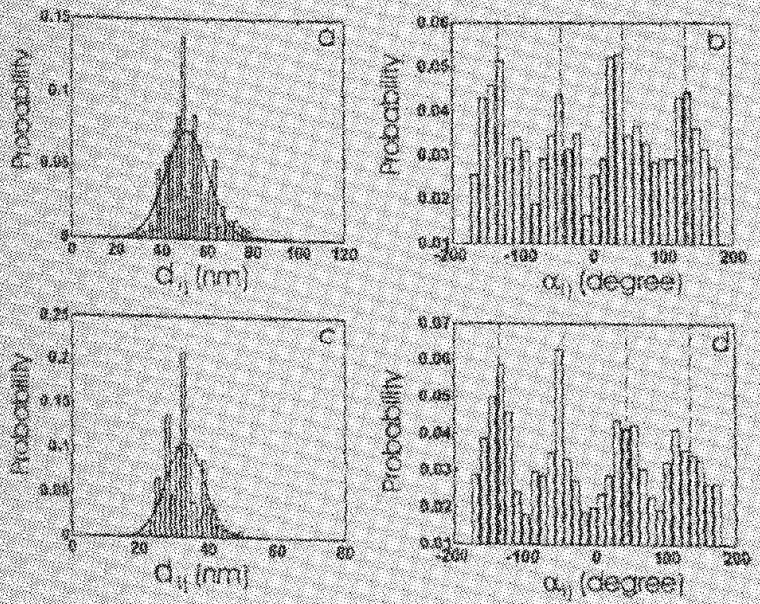

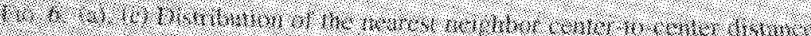

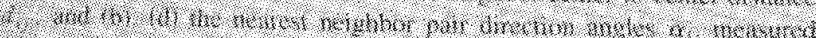

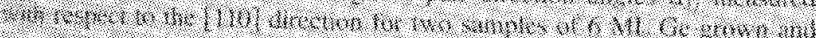

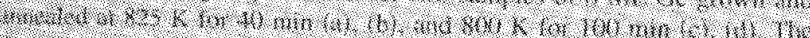

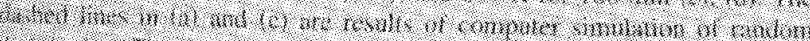

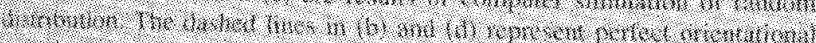

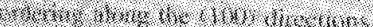

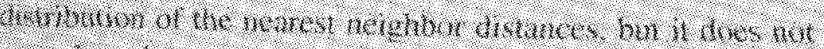

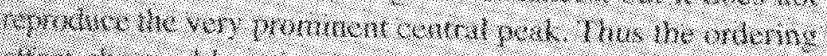

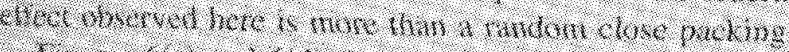

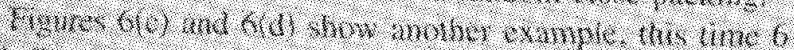

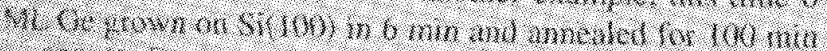

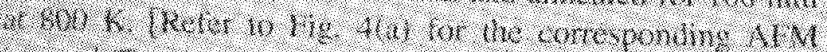

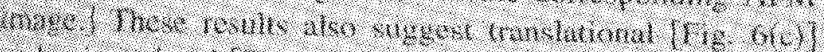

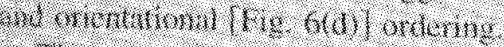

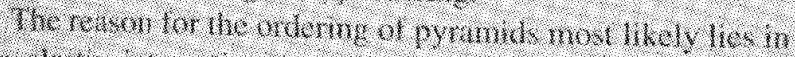

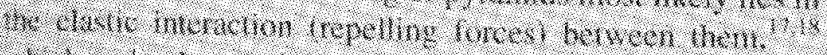

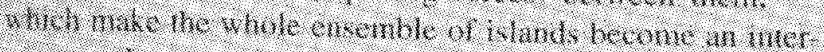

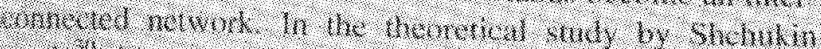

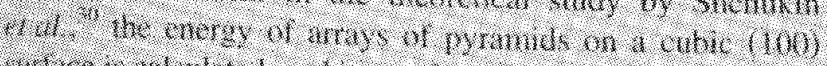

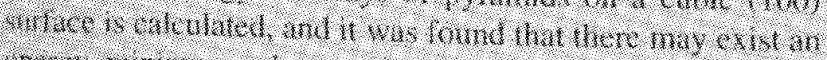

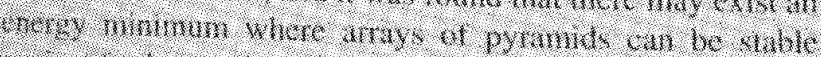

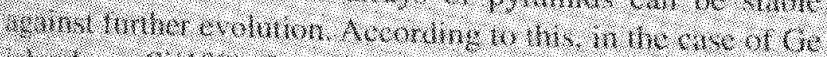

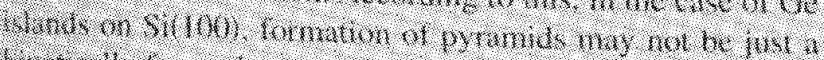

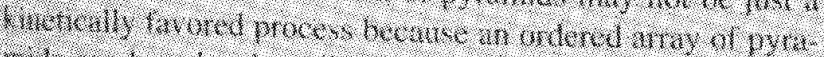

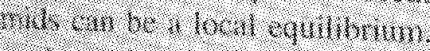

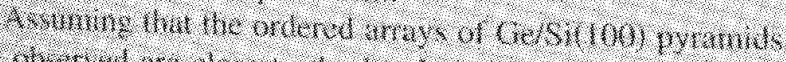

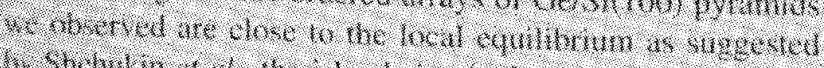

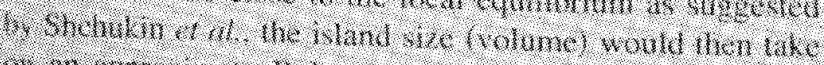

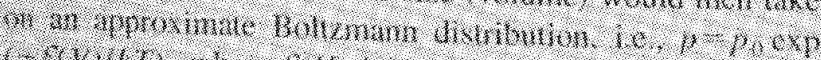

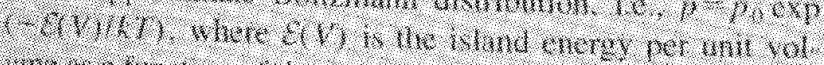

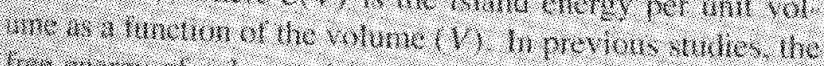
pro migr.

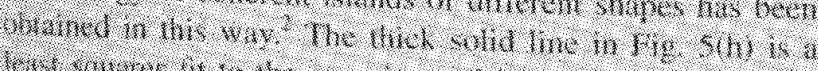

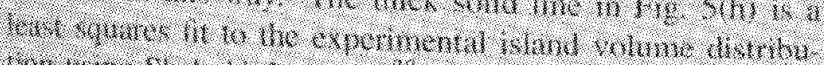

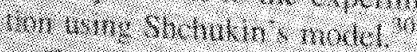

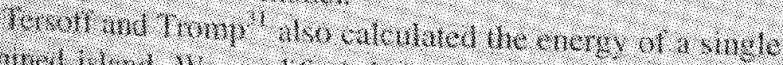

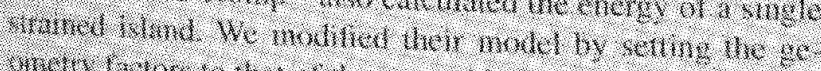

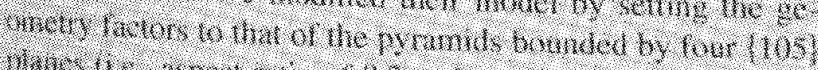

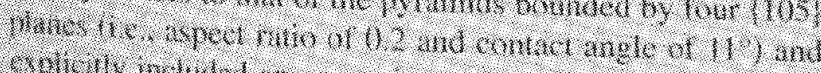

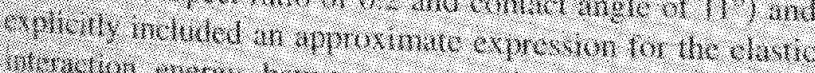

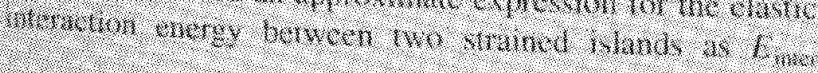

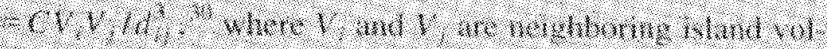

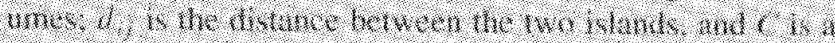

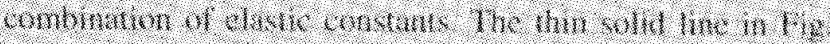

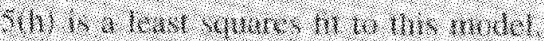

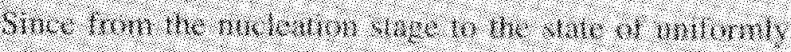

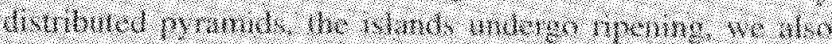

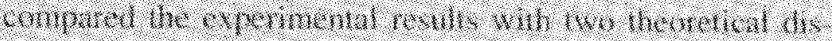

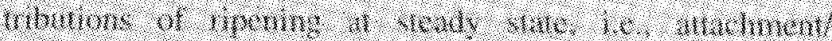

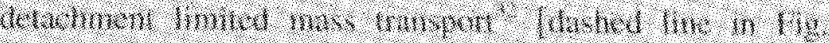

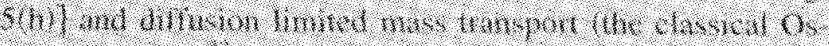

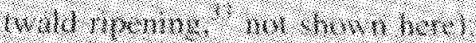

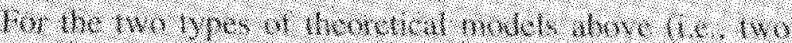

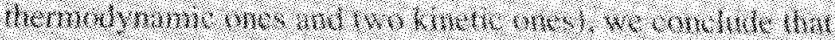

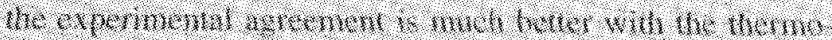

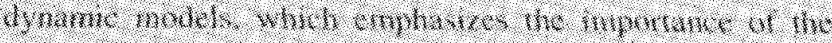

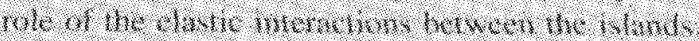

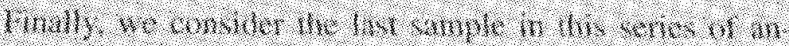

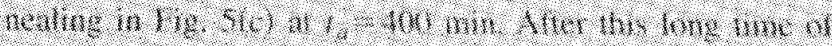

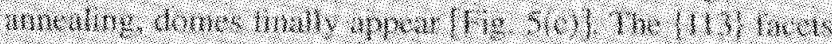

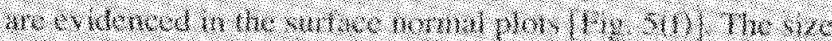

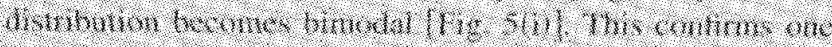

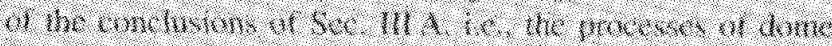

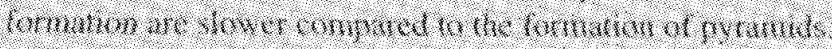

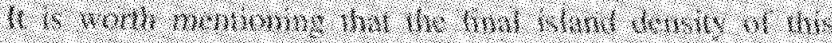

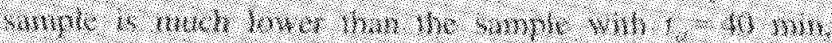

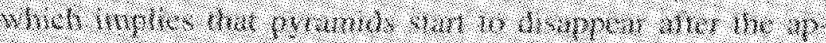

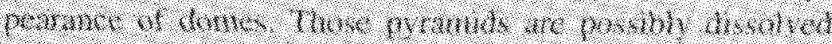

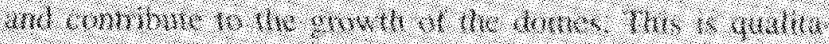

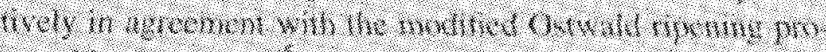

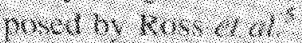

\section{N. Concu USIONS}

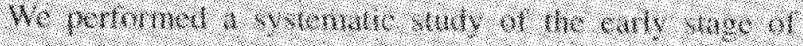

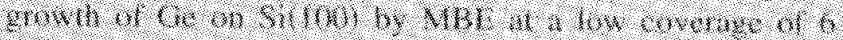

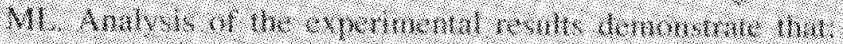

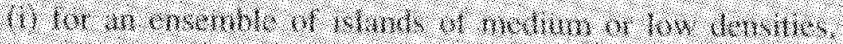

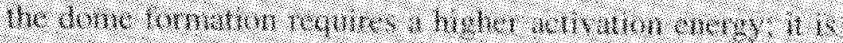

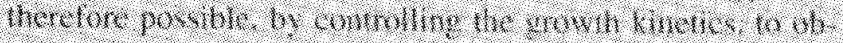

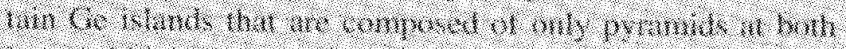

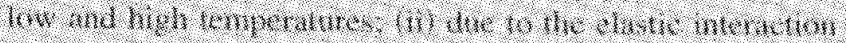

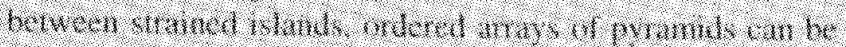

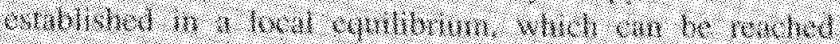

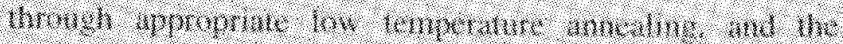

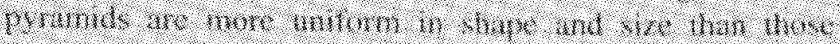

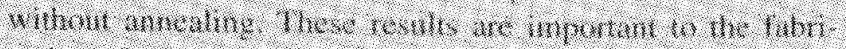

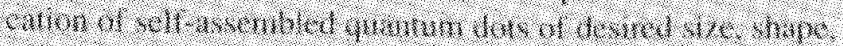

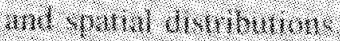

\section{ACKNOWLEDCWENTS}

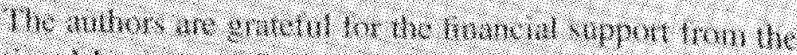

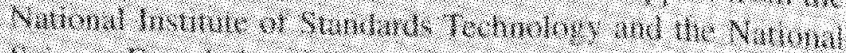

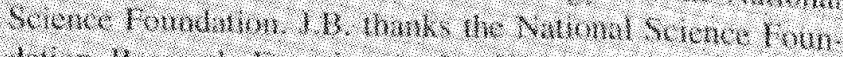

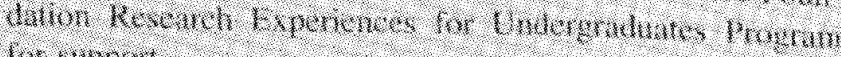

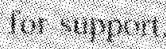




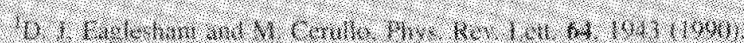

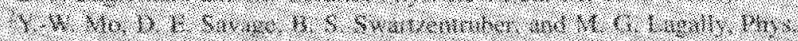

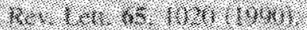

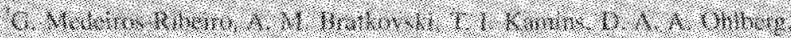

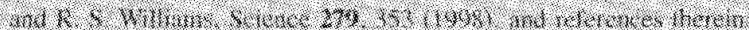

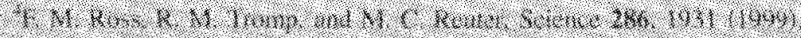

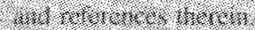

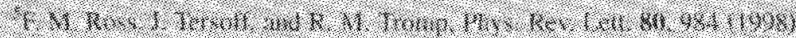

.

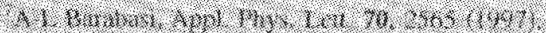

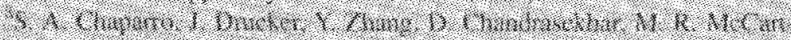

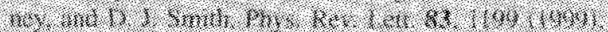

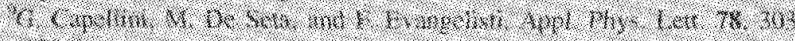
$+1+1$

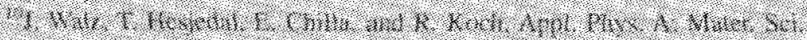

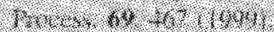

4.

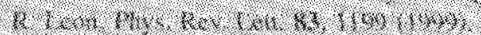

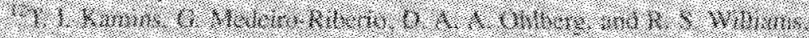
, N.

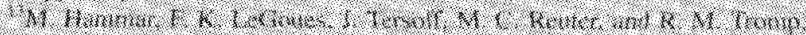

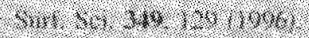

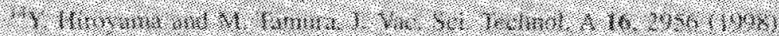

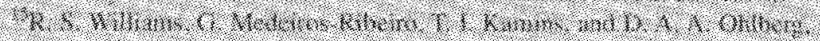

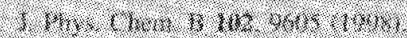

r.

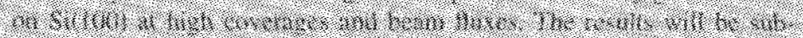

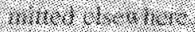

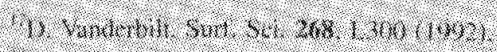

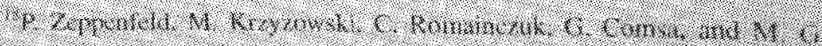
1.

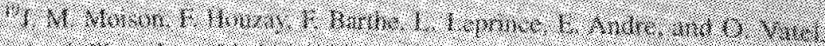

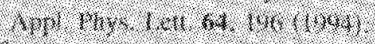

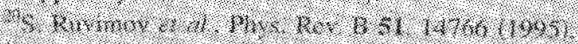

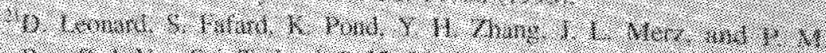

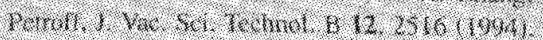

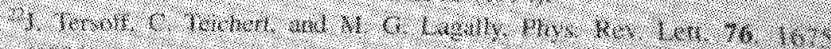
(1)

r.

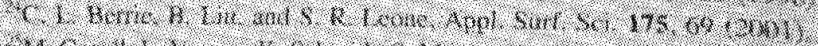

,

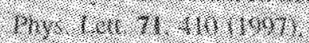

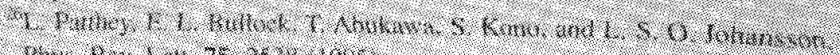

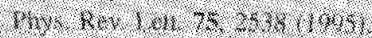

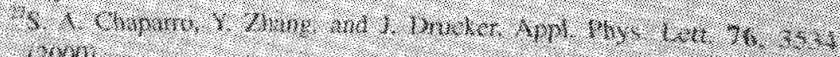

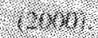

- r.

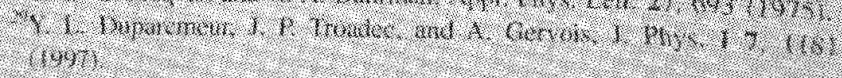

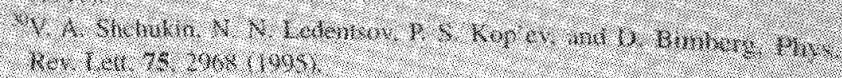

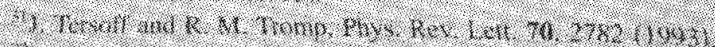

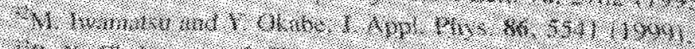

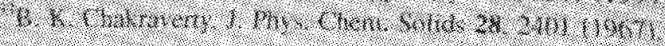

